\title{
Input Admittance, Directivity and Quality Factor of Biconical Antenna of Arbitrary Cone Angle
}

This paper was downloaded from TechRxiv (https://www.techrxiv.org).

\section{LICENSE}

CC BY 4.0

SUBMISSION DATE / POSTED DATE

$21-07-2021 / 26-07-2021$

\section{CITATION}

Janaswamy, Ramakrishna (2021): Input Admittance, Directivity and Quality Factor of Biconical Antenna of Arbitrary Cone Angle. TechRxiv. Preprint. https://doi.org/10.36227/techrxiv.15032082.v1

$\mathrm{DOI}$ 


\title{
Input Admittance, Directivity and Quality Factor of Biconical Antenna of Arbitrary Cone Angle
}

\author{
Ramakrishna Janaswamy, Fellow, IEEE
}

\begin{abstract}
New analytical expressions and numerical results for the quality factor and directivity as well as computationally convenient expressions for the input admittance of a symmetrical biconical antenna of arbitrary length $L$ and cone angle $\theta_{0}$ are presented. The quality factor for a wide-angle biconical antenna is shown to very closely approach the Chu's limit of $Q=(k L)^{-1}\left\{1+(k L)^{-2}\right\}$. Numerical calculations based on the analytical formula for antenna admittance confirm the conjecture that Foster's reactance theorem remains invalid even for perfectly conducting antennas. Furthermore, the variation of directivity of a wide-angle biconical antenna is a slowly varying function of its electrical length and is shown to depart significantly from that of a thin cylindrical dipole.
\end{abstract}

Index Terms-Biconical antenna, analytical methods, associated Legendre function, quality factor, input impedance, directivity.

\section{INTRODUCTION}

$\mathbf{T}$ HE biconical antenna is one of the few finite-sized structures that is amenable to treatment by analytical methods. Furthermore, owing to its tapered shape, see Fig. 1. it provides relatively wide bandwidths of operation as well as present input impedance values that permit direct connection to practical feed lines. The antenna has previously been analyzed extensively by Schelkunoff [1] and later by Tai [2] and these works are summarized in [3], [4] and [5]. Numerical data are available in the literature for small cone angles $\left(\theta_{0} \ll 1\right)$ as well for large cone angles $\left(\theta_{0} \rightarrow \pi / 2\right)$. The former is a good model for a thin cylindrical dipole and the latter to a spherical antenna with a thin equatorial slot. These two special cases not only reduce to elementary geometries but also permit analytical approximations that have been taken advantage of by earlier workers. Based on these approximations, input impedance and radiation patterns of wide-angle biconical antenna were studied in [6] and [7]. Bevensee [8] generates extensive numerical data for input impedance and radiation patterns for cone angles up to 45 degrees. Mayes [9] conducts experimental studies on tunable, wide-angle monopoles with loaded lumped elements to achieve specified bandwidth. Biconical antenna with unequal cone angles has been treated in [10] and numerical results for antenna patterns were provided once again only for small and wide cone angles.

Notwithstanding the above earlier works, given the practical importance of the antenna in a variety of wideband applications, including for calibration purposes [11] and its potential to serve as a benchmark antenna for computational methods

R. Janaswamy is with the Department of Electrical and Computer Engineering, University of Massachusetts, Amherst, MA, 01003 USA e-mail: janaswam@umass.edu.
[12], it is desirable to have detailed analytical steps leading to the generation of numerical data for various properties of the biconical antenna with arbitrary structural parameters. The goal of the present paper is to provide this much-needed information and fill the void that exists in the literature. Modern texts on antennas such as [13][p. 213], [14][p. 500], [15] treat the finite-sized biconical antenna only peripherally, while dismissing the analysis as being too complicated. Exact expressions and numerical procedures are presented below for the general case and numerical data are generated for $\theta_{0}=\pi / 4$. Of particular interest here are convenient expression for the input admittance and new expressions for the quality factor and the directivity of biconical antenna for arbitrary cone angles and electrical lengths.

\section{THEORY}

\section{A. Field Expressions and Basic Antenna Properties}

A symmetric, coaxial biconical antenna having identical perfectly conducting cones with half angles $\theta_{0}$ and axes aligned is shown in Fig. 1. The region between the two cones is defined by $\theta_{0} \leq \theta \leq \pi-\theta_{0}, 0<r \leq L$. An infinitesimal source is assumed to be placed at the apex of the antenna.

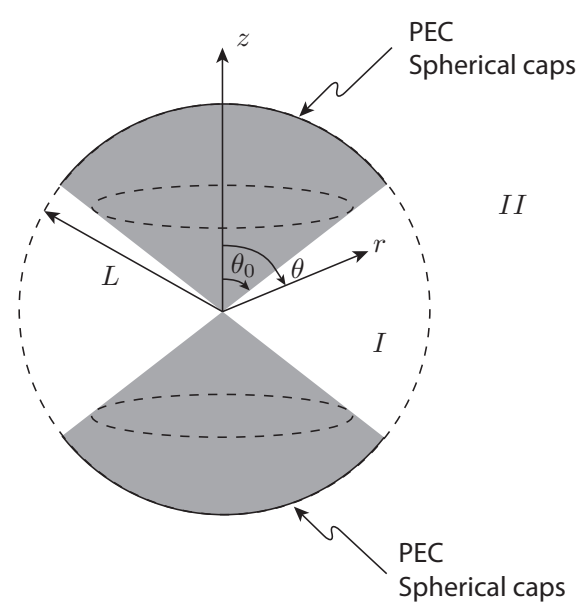

Fig. 1. Symmetrical biconical antenna having radius $L$ and half-angle $\theta_{0}$. The cones are sealed off with perfectly conducting spherical caps.

We assume operation in a balanced mode where the feed electric field present at the apex is an even function of polar angle $\theta$. The only non-zero components of field in such a case are $E_{r}, E_{\theta}$, and $H_{\phi}$ and these are all azimuthally invariant. Such a field constitutes low order $\mathrm{TM}_{r}$ modes, that is, those 
having no azimuthal variation. The input admittance, $Y_{\text {in }}$, of the antenna is defined as

$$
Y_{\text {in }}=\frac{I_{0}}{V_{0}}:=\lim _{r \rightarrow 0} \frac{2 \pi r \sin \theta H_{\phi}}{\int_{\theta_{0}}^{\pi-\theta_{0}} r E_{\theta} d \theta} .
$$

For $\mathrm{TM}_{r}$ modes, the complete fields can be obtained in terms of the radial electric field by an application of BouwkampCasimir theorem [16, p. 1-29].

For an $e^{j \omega t}$ time convention at the angular frequency $\omega$, the fields in region-I (that is, for $r<L$ ) are expressed as

$$
\begin{aligned}
r E_{r}= & -\frac{j \eta I_{0}}{2 \pi k r} \sum_{\nu_{n}} a_{\nu_{n}} \frac{\widehat{J}_{\nu_{n}}(k r)}{\widehat{J}_{\nu_{n}}(k L)} M_{\nu_{n}}(\theta) \\
r E_{\theta}= & -\frac{j \eta I_{0}}{2 \pi} \sum_{\nu_{n}} \frac{a_{\nu_{n}}}{\nu_{n}\left(\nu_{n}+1\right)} \frac{\widehat{J}_{\nu_{n}}^{\prime}(k r)}{\widehat{J}_{\nu_{n}}(k L)} \frac{\partial M_{\nu_{n}}(\theta)}{\partial \theta} \\
& +r E_{0}(r, \theta) \\
r H_{\phi}= & -\frac{I_{0}}{2 \pi} \sum_{\nu_{n}} \frac{a_{\nu_{n}}}{\nu_{n}\left(\nu_{n}+1\right)} \frac{\widehat{J}_{\nu_{n}}(k r)}{\widehat{J}_{\nu_{n}}(k L)} \frac{\partial M_{\nu_{n}}(\theta)}{\partial \theta} \\
& +r H_{0}(r, \theta),
\end{aligned}
$$

where $\widehat{J}_{\nu_{n}}(\cdot), \widehat{H}_{\nu_{n}}^{(2)}(\cdot)$ are spherical Ricatti-Bessel and Hankel functions, respectively, of order, $\nu_{n}, M_{\nu_{n}}(\theta)=\left[P_{\nu_{n}}(\cos \theta)-\right.$ $\left.P_{\nu_{n}}(-\cos \theta)\right] / 2$ is an odd function of $\theta$ about $\theta=\pi / 2, P_{\nu}(\cdot)$ is the Legendre function of degree $\nu, \eta=\sqrt{\mu_{0} / \epsilon_{0}}$ is the intrinsic impedance and $k=\omega \sqrt{\mu_{0} \epsilon_{0}}$ is the wavenumber in free-space. A prime on Bessel functions denotes derivative with respect to the argument.

The fields $E_{0}, H_{0}$ correspond to outgoing and incoming radial TEM modes and are assumed to be of the form

$$
\begin{aligned}
r E_{0}(r, \theta)= & \frac{I_{0} \eta}{4 \pi \sin \theta}\left[\left(1+K Y_{t}\right) e^{-j k(r-L)}\right. \\
& \left.+\left(1-K Y_{t}\right) e^{j k(r-L)}\right] \\
r H_{0}(r, \theta)= & \frac{I_{0}}{4 \pi \sin \theta}\left[\left(1+K Y_{t}\right) e^{-j k(r-L)}\right. \\
& \left.-\left(1-K Y_{t}\right) e^{j k(r-L)}\right] .
\end{aligned}
$$

The constant $K$ is the characteristic impedance of an infinite biconical transmission line and is related to the half-angle of the cone via

$$
K=\frac{\eta}{2 \pi} \int_{\theta_{0}}^{\pi-\theta_{0}} \frac{1}{\sin \theta} d \theta=\frac{\eta}{\pi} \ln \left[\cot \left(\frac{\theta_{0}}{2}\right)\right],
$$

and the constant $Y_{t}$ is the normalized terminal admittance such that

$$
K Y_{t}=\frac{\eta H_{0}(L, \theta)}{E_{0}(L, \theta)} .
$$

From the field expressions of $E_{0}$ and $H_{0}$ it is clear that $2 \pi r \sin \theta E_{0}(L, \theta)=I_{0} \eta$ and $2 \pi r \sin \theta H_{0}(L, \theta)=I_{0} K Y_{t}$ so that $(8)$ follows directly from these.

In order that $E_{r}=0$ on the conical surfaces at $\theta=\theta_{0}, \pi-$ $\theta_{0}$, the order $\nu_{n}$ must be a root of $M_{\nu_{n}}\left(\theta_{0}\right)=0$, that is, of

$$
P_{\nu_{n}}\left(\cos \theta_{0}\right)-P_{\nu_{n}}\left(-\cos \theta_{0}\right)=0, n=1,2, \ldots
$$

Clearly, the distribution of roots depends only on the halfangle $\theta_{0}$ of the biconical antenna. Equation (9) will have a countably infinite number of roots $\nu_{n}>0$ as can be easily ascertained from the asymptotic form of Legendre function [17. p. 419] subject to $q=\sqrt{\left(\nu_{n}+\frac{1}{2}\right)^{2}+\frac{1}{4}} \gg 1$

$$
\begin{aligned}
M_{\nu_{n}}(\theta) \sim & \sqrt{\frac{2}{\pi \sin \theta \sqrt{\nu_{n}\left(\nu_{n}+1\right)}}} \sin \left(\frac{q \pi}{2}-\frac{\pi}{4}\right) \\
& \sin \left[q\left(\frac{\pi}{2}-\theta\right)\right] .
\end{aligned}
$$

Using this asymptotic form, the non-integer zeros of the function $M_{\nu_{n}}\left(\theta_{0}\right)$ and the corresponding derivatives are easily determined as

$$
\begin{aligned}
\nu_{n} & \sim \sqrt{n^{2} \alpha_{0}^{2}-\frac{1}{4}}-0.5 \sim n \alpha_{0}-0.5 \\
\frac{d \nu_{n}}{d \theta_{0}} & \sim \frac{n^{2} \alpha_{0}^{3}}{\pi\left(\nu_{n}+\frac{1}{2}\right)} \sim \frac{n \alpha_{0}^{2}}{\pi}, n=1,2, \ldots
\end{aligned}
$$

where $\alpha_{0}=\pi /\left(\pi / 2-\theta_{0}\right)$. For a cone half-angle of $\theta_{0}=\pi / 4$, the slopes of linear trends in equations (11) and $(12)$ for $\nu_{n}$ and $d \nu_{n} / d \theta_{0}$ are respectively, $\alpha_{0}=4$ and $\alpha_{0}^{2} / \pi \approx 5.0930$.

The Legendre function $M_{\nu_{n}}(\theta)$ can also be expressed in terms of a hypergeometric function $F(\cdot)$ and the Gamma function $\Gamma(\cdot)$ as [18, p. 178]

$$
\begin{aligned}
M_{\nu_{n}}(\theta)= & \frac{2 \Gamma\left(\frac{\nu_{n}}{2}+1\right)}{\sqrt{\pi} \Gamma\left(\frac{\nu_{n}}{2}+\frac{1}{2}\right)} \sin \left(\frac{\nu_{n} \pi}{2}\right) \cos \theta \\
& \times F\left(\frac{1}{2}-\frac{\nu_{n}}{2}, \frac{\nu_{n}}{2}+1 ; \frac{3}{2} ; \cos ^{2} \theta\right),
\end{aligned}
$$

so that $\nu_{n}$ is also a root of

$$
F\left(\frac{1}{2}-\frac{\nu_{n}}{2}, \frac{\nu_{n}}{2}+1 ; \frac{3}{2} ; \cos ^{2} \theta_{0}\right)=0, n=1,2, \ldots
$$

The roots $\nu_{n}$ of the equation (14) for a given value of $\theta_{0}$ can be calculated numerically by generating the hypergeometric function $F(\cdot)$ from its governing differential equation [19. Equation 9.151]. The derivative $d \nu_{n} / d \theta_{0}$ can also be calculated numerically using the difference formula $d \nu_{n} / d \theta_{0} \approx$ $\left[\nu_{n}\left(\theta_{0}+\delta \theta_{0}\right)-\nu_{n}\left(\theta_{0}\right)\right] / \delta \theta_{0}$. Table I shows the first thirty roots and the associated derivatives for $\theta_{0}=\pi / 4$ using $\delta \theta_{0}=5 \times 10^{-4} \mathrm{rad}$. These numerical generated values from equation (14) are compared with the asymptotic values given in (11) and (12). Figure 2 shows a plot of the roots $\nu_{n}$ and the derivative $d \nu_{n} / d \theta_{0}$ versus $n$ for the numerically and asymptotically generated values. The discrete data are joined by a smooth curve for visual clarity. Excellent agreement is seen between the two sets of data. A linear trend is clearly seen for both $\nu_{n}$ and $d \nu_{n} / d \theta_{0}$ with $n$. The numerically calculated values of $\nu_{n}$ and $d \nu_{n} / d \theta_{0}$ can be fit by linear regression to result in

$\nu_{n}=4.0005 n-0.5135 ; \frac{d \nu_{n}}{d \theta_{0}}=5.0954 n+2.8012 \times 10^{-2}$.

Asymptotic formula based values for $\nu_{n}$ and $d \nu_{n} / d \theta_{0}$ given after equation (12) are in excellent agreement with the numerically generated ones in 15 and the absolute error is less 
than $1.25 \times 10^{-2} \%$ and $4.8 \times 10^{-2} \%$, respectively. Clearly, the asymptotic values are already very accurate (less than $0.2 \%$ error) even for $n=1$ and get increasingly better for higher $n$. The asymptotic formulas (11) and (12) are also effective for very low cone angles. For instance, for the extremely small value of $\theta_{0}=1^{\circ}$, the actual root $\nu_{1}=1.26$ whereas that predicted by formula $\left[11\right.$ is $\nu_{1} \sim 1.46$. Accuracy will only increase as the root order increases.

TABLE I

First thirty roots of equation 14 for $\theta_{0}=\frac{\pi}{4}$. determined by an application of the boundary conditions at $r=L$, which will be elaborated upon below. The input admittance, $Y_{\mathrm{in}}$, of the antenna is defined as the ratio of apex current to the voltage difference between the two cones

$$
Y_{\text {in }}=\lim _{r \rightarrow 0} \frac{2 \pi r \sin \theta H_{\phi}}{\int_{\theta_{0}}^{\pi-\theta_{0}} r E_{\theta} d \theta}=\frac{1}{K} \frac{1-\Gamma_{L}}{1+\Gamma_{L}},
$$

where $\Gamma_{L}$ is the reflection coefficient at $r=L$ of the TEM mode ${ }^{1}$ and equals

$$
\Gamma_{L}=\frac{1-K Y_{t}}{1+K Y_{t}} e^{-2 j k L}
$$

\begin{tabular}{|c|c|c|c|c|}
\hline Index $n$ & Numerical $\nu_{n}$ & Numerical $\frac{d \nu_{n}}{d \theta_{0}}$ & Asymptotic $\nu_{n}$ & Asymptotic $\frac{d \nu_{n}}{d \theta_{0}}$ \\
\hline 1 & $3.4620574 \mathrm{e}+00$ & $5.2057059 \mathrm{e}+00$ & $3.4686270 \mathrm{e}+00$ & $5.1332193 \mathrm{e}+00$ \\
\hline 2 & $7.4804257 \mathrm{e}+00$ & $1.0220051 \mathrm{e}+01$ & $7.4843597 \mathrm{e}+00$ & $1.0205869 \mathrm{e}+01$ \\
\hline 3 & $1.1486850 \mathrm{e}+01$ & $1.5285263 \mathrm{e}+01$ & $1.1489579 \mathrm{e}+01$ & $1.5292155 \mathrm{e}+01$ \\
\hline 4 & $1.5490107 \mathrm{e}+01$ & $2.0368186 \mathrm{e}+01$ & $1.5492186 \mathrm{e}+01$ & $2.0381787 \mathrm{e}+01$ \\
\hline 5 & $1.9492073 \mathrm{e}+01$ & $2.5490328 \mathrm{e}+01$ & $1.9493749 \mathrm{e}+01$ & $2.5472752 \mathrm{e}+01$ \\
\hline 6 & $2.3493388 \mathrm{e}+01$ & $3.0597680 \mathrm{e}+01$ & $2.3494791 \mathrm{e}+01$ & $3.0564383 \mathrm{e}+01$ \\
\hline 7 & $2.7494329 \mathrm{e}+01$ & $3.5698304 \mathrm{e}+01$ & $2.7495535 \mathrm{e}+01$ & $3.5656393 \mathrm{e}+01$ \\
\hline 8 & $3.1495036 \mathrm{e}+01$ & $4.0795498 \mathrm{e}+01$ & $3.1496094 \mathrm{e}+01$ & $4.0748640 \mathrm{e}+01$ \\
\hline 9 & $3.5495586 \mathrm{e}+01$ & $4.5890810 \mathrm{e}+01$ & $3.5496528 \mathrm{e}+01$ & $4.5841045 \mathrm{e}+01$ \\
\hline 10 & $3.9496027 \mathrm{e}+01$ & $5.0985049 \mathrm{e}+01$ & $3.9496875 \mathrm{e}+01$ & $5.0933561 \mathrm{e}+01$ \\
\hline 11 & $4.3496387 \mathrm{e}+01$ & $5.6078677 \mathrm{e}+01$ & $4.3497159 \mathrm{e}+01$ & $5.6026157 \mathrm{e}+01$ \\
\hline 12 & $4.7496688 \mathrm{e}+01$ & $6.1171979 \mathrm{e}+01$ & $4.7497396 \mathrm{e}+01$ & $6.1118814 \mathrm{e}+01$ \\
\hline 13 & $5.1496942 \mathrm{e}+01$ & $6.6265145 \mathrm{e}+01$ & $5.1497596 \mathrm{e}+01$ & $6.6211517 \mathrm{e}+01$ \\
\hline 14 & $5.5497160 \mathrm{e}+01$ & $7.1358308 \mathrm{e}+01$ & $5.5497768 \mathrm{e}+01$ & $7.1304257 \mathrm{e}+01$ \\
\hline 15 & $5.9497349 \mathrm{e}+01$ & $7.6451570 \mathrm{e}+01$ & $5.9497917 \mathrm{e}+01$ & $7.6397025 \mathrm{e}+01$ \\
\hline 16 & $6.3497515 \mathrm{e}+01$ & $8.1545013 \mathrm{e}+01$ & $6.3498047 \mathrm{e}+01$ & $8.1489818 \mathrm{e}+01$ \\
\hline 17 & $6.7497661 \mathrm{e}+01$ & $8.6638706 \mathrm{e}+01$ & $6.7498162 \mathrm{e}+01$ & $8.6582630 \mathrm{e}+01$ \\
\hline 18 & $7.1497791 \mathrm{e}+01$ & $9.1737112 \mathrm{e}+01$ & $7.1498264 \mathrm{e}+01$ & $9.1675458 \mathrm{e}+01$ \\
\hline 19 & $7.5497907 \mathrm{e}+01$ & $9.6836166 \mathrm{e}+01$ & $7.5498355 \mathrm{e}+01$ & $9.6768300 \mathrm{e}+01$ \\
\hline 20 & $7.9498012 \mathrm{e}+01$ & $1.0193433 \mathrm{e}+02$ & $7.9498437 \mathrm{e}+01$ & $1.0186115 \mathrm{e}+02$ \\
\hline 21 & $8.3498106 \mathrm{e}+01$ & $1.0703175 \mathrm{e}+02$ & $8.3498512 \mathrm{e}+01$ & $1.0695402 \mathrm{e}+02$ \\
\hline 22 & $8.7498192 \mathrm{e}+01$ & $1.1212855 \mathrm{e}+02$ & $8.7498580 \mathrm{e}+01$ & $1.1204689 \mathrm{e}+02$ \\
\hline 23 & $9.1498271 \mathrm{e}+01$ & $1.1722483 \mathrm{e}+02$ & $9.1498641 \mathrm{e}+01$ & $1.1713977 \mathrm{e}+02$ \\
\hline 24 & $9.5498343 e+01$ & $1.2232069 \mathrm{e}+02$ & $9.5498698 \mathrm{e}+01$ & $1.2223265 \mathrm{e}+02$ \\
\hline 25 & $9.9498409 \mathrm{e}+01$ & $1.2741621 \mathrm{e}+02$ & $9.9498750 \mathrm{e}+01$ & $1.2732555 \mathrm{e}+02$ \\
\hline 26 & $1.0349847 \mathrm{e}+02$ & $1.3251148 \mathrm{e}+02$ & $1.0349880 \mathrm{e}+02$ & $1.3241844 \mathrm{e}+02$ \\
\hline 27 & $1.0749853 \mathrm{e}+02$ & $1.3760657 \mathrm{e}+02$ & $1.0749884 \mathrm{e}+02$ & $1.3751134 \mathrm{e}+02$ \\
\hline 28 & $1.1149858 \mathrm{e}+02$ & $1.4270155 \mathrm{e}+02$ & $1.1149888 \mathrm{e}+02$ & $1.4260425 \mathrm{e}+02$ \\
\hline 29 & $1.1549863 \mathrm{e}+02$ & $1.4779648 \mathrm{e}+02$ & $1.1549892 \mathrm{e}+02$ & $1.4769716 \mathrm{e}+02$ \\
\hline 30 & $1.1949867 \mathrm{e}+02$ & $1.5289142 \mathrm{e}+02$ & $1.1949896 \mathrm{e}+02$ & $1.5279007 \mathrm{e}+02$ \\
\hline
\end{tabular}

The fields in region-II (that is, for $r>L$ ) are expressed as

$$
\begin{aligned}
& r E_{r}=\frac{-j \eta I_{0}}{2 \pi k r} \sum_{\ell=1,3}^{\infty} \ell(\ell+1) c_{\ell} \frac{\widehat{H}_{\ell}^{(2)}(k r)}{\widehat{H}_{\ell}^{(2)}(k L)} P_{\ell}(\cos \theta)(18 \\
& r E_{\theta}=-\frac{j I_{0} \eta}{2 \pi} \sum_{\ell=1,3}^{\infty} c_{\ell} \frac{\widehat{H}_{\ell}^{(2)^{\prime}}(k r)}{\widehat{H}_{\ell}^{(2)}(k L)} \frac{d P_{\ell}(\cos \theta)}{d \theta} \\
& r H_{\phi}=-\frac{I_{0}}{2 \pi} \sum_{\ell=1,3}^{\infty} c_{\ell} \frac{\widehat{H}_{\ell}^{(2)}(k r)}{\widehat{H}_{\ell}^{(2)}(k L)} \frac{d P_{\ell}(\cos \theta)}{d \theta}
\end{aligned}
$$

where $P_{\ell}(\cos \theta)$ is a Legendre function of first kind of integer degree $\ell$ and $c_{\ell}$ are the mode constants to be determined by an enforcement of boundary conditions at $r=L$. As with the interior region, the fields $E_{\theta}$ and $H_{\phi}$ are both even functions of $\theta$ about $\theta=\pi / 2$, while $E_{r}$ is an odd function. Bouwkamp and Casimir's theorem guarantees that there will be no TEM waves in region-II, so the expansions given in $(18)-(20)$ are complete for region-II.

The far-zone fields are obtained by setting $\widehat{H}_{\ell}^{(2)^{\prime}}(k r) \sim$ $j^{\ell+1} e^{-j k r}$, resulting in

$$
r E_{\theta}=\eta r H_{\phi} \sim \frac{I_{0} \eta e^{-j k r}}{2 \pi} \sum_{\ell=1,3}^{\infty} \frac{c_{\ell} j^{\ell}}{\widehat{H}_{\ell}^{(2)}(k L)} \frac{d P_{\ell}(\cos \theta)}{d \theta} .
$$

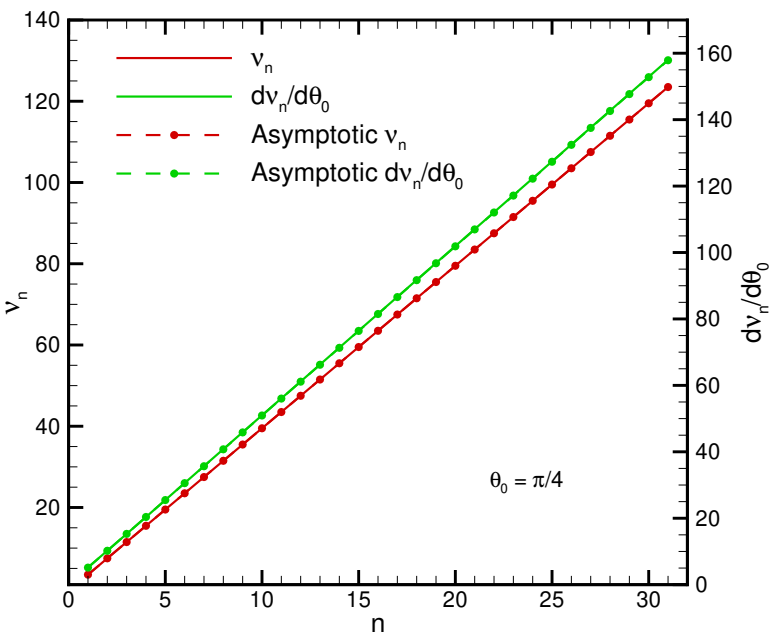

Fig. 2. First thirty one roots of equation 14 and zeros of 10 .

The remaining unknown coefficients $a_{\nu_{n}}$ in (2)-(4) are
By matching the fields $E_{\theta}$ and $H_{\phi}$ at $r=L$ the following relations between the expansion coefficients $c_{\ell}$ and $a_{\nu_{n}}$ are obtained

$$
\sum_{\ell=1,3}^{\infty} c_{\ell} \frac{d P_{\ell}(\cos \theta)}{d \theta}= \begin{cases}\sum_{n=1}^{\infty} \frac{a_{\nu_{n}} \frac{\partial M_{\nu_{n}}}{\nu_{n}\left(\nu_{n}+1\right)}-\frac{K Y_{t}}{\sin \theta},}{\theta_{0}<\theta<\pi-\theta_{0}} \\ 0, & \text { otherwise }\end{cases}
$$

and

$$
\begin{gathered}
\sum_{\ell=1,3}^{\infty} c_{\ell} \frac{\widehat{H}_{\ell}^{(2) \prime}(k L)}{\widehat{H}_{\ell}^{(2)}(k L)} \frac{d P_{\ell}(\cos \theta)}{d \theta}= \\
\begin{cases}\sum_{n=1}^{\infty} \frac{a_{\nu_{n}} \frac{\widehat{J}_{\nu_{n}}^{\prime}(k L)}{\widehat{J}_{\nu_{n}}(k L)} \frac{\partial M_{\nu_{n}}}{\partial \theta}}{\nu_{n}\left(\nu_{n}+1\right)}+\frac{j}{\sin \theta}, & \theta_{0}<\theta<\pi-\theta_{0} \\
0, & \text { otherwise }\end{cases}
\end{gathered}
$$

${ }^{1}$ Note that the non-TEM parts of the magnetic and electric fields in region-I do not contribute to the current and voltage difference at the apex. 
The following integral relations are easy to establish based on the governing differential equation for Legendre function [16, p. D-15]:

$$
\begin{aligned}
L_{\ell, n} & =\int_{0}^{\pi} P_{\ell}(\cos \theta) P_{n}(\cos \theta) \sin \theta d \theta \\
& =\frac{2}{2 n+1} \delta_{\ell}^{n} \\
L_{\ell, n}^{\prime} & =\int_{0}^{\pi} \frac{d P_{\ell}(\cos \theta)}{d \theta} \frac{d P_{n}(\cos \theta)}{d \theta} \sin \theta d \theta \\
& =\frac{2 n(n+1)}{2 n+1} \delta_{\ell}^{n} \\
T_{\ell, \nu_{n}}\left(\theta_{0}\right) & =\frac{1}{2} \int_{\theta_{0}}^{\pi-\theta_{0}} P_{\ell}(\cos \theta) M_{\nu_{n}}(\theta) \sin \theta d \theta \\
& =\frac{\left.\sin \theta_{0} P_{\ell}\left(\cos \theta_{0}\right) \frac{\partial M_{\nu_{n}}}{\partial \theta}\right|_{\theta_{0}}}{\nu_{n}\left(\nu_{n}+1\right)-\ell(\ell+1)} \\
I_{\nu_{n}, \nu_{m}}\left(\theta_{0}\right) & =\frac{1}{2} \int_{\theta_{0}}^{\pi-\theta_{0}} \frac{\partial M_{\nu_{m}}(\theta)}{\partial \theta} \frac{\partial M_{\nu_{n}}(\theta)}{\partial \theta} \sin \theta d \theta \\
& =\frac{\nu_{n}\left(\nu_{n}+1\right)}{2} \int_{\theta_{0}}^{\pi-\theta_{0}} M_{\nu_{m}}(\theta) M_{\nu_{n}}(\theta) \sin \theta d \theta(28) \\
& =\delta_{n}^{m} \frac{\nu_{n}\left(\nu_{n}+1\right)}{\left(2 \nu_{n}+1\right)} \sin \theta_{0}\left[\frac{\partial M_{\nu_{n}}}{\partial \theta}\right]^{2}\left[\frac{d \nu_{n}}{d \theta}\right]^{-1},
\end{aligned}
$$

where $\delta_{n}^{m}$ is the Kronecker's delta equal to 1 if $m=n$ and zero otherwise. Making use of these relations in appropriate integrals with respect to $\theta$ of (22) and (23) and carrying out several algebraic manipulations we are led to

$$
\begin{gathered}
K Y_{t}=\frac{1}{\ln \left[\cot \left(\frac{\theta_{0}}{2}\right)\right]} \sum_{\ell=1,3}^{\infty} c_{\ell} P_{\ell}\left(\cos \theta_{0}\right), \\
a_{\nu_{n}}=\frac{\nu_{n}\left(\nu_{n}+1\right)}{I_{\nu_{n}, \nu_{n}}\left(\theta_{0}\right)} \sum_{\ell=1,3}^{\infty} \ell(\ell+1) c_{\ell} T_{\ell, \nu_{n}}\left(\theta_{0}\right),
\end{gathered}
$$

and

$$
\begin{aligned}
& \sum_{\ell=1,3}^{\infty}\left[m(m+1) \ell(\ell+1) \sin \theta_{0} P_{m}\left(\cos \theta_{0}\right) P_{\ell}\left(\cos \theta_{0}\right) g_{m \ell}\right. \\
& \left.-\frac{m(m+1)}{(2 m+1)} \frac{\widehat{H}_{m}^{(2)^{\prime}}(k L)}{\widehat{H}_{m}^{(2)}(k L)} \delta_{m}^{\ell}\right] c_{\ell}=j P_{m}\left(\cos \theta_{0}\right),
\end{aligned}
$$

where

$$
\begin{aligned}
g_{m \ell}= & g_{\ell m}=\sum_{n=1}^{\infty} \frac{\left(2 \nu_{n}+1\right)}{\nu_{n}\left(\nu_{n}+1\right)} \frac{\widehat{J}_{\nu_{n}}^{\prime}(k L)}{\widehat{J}_{\nu_{n}}(k L)} \frac{d \nu_{n}}{d \theta_{0}} \times \\
& \frac{1}{\left[\nu_{n}\left(\nu_{n}+1\right)-m(m+1)\right]\left[\nu_{n}\left(\nu_{n}+1\right)-\ell(\ell+1)\right]} .
\end{aligned}
$$

Equation (31) is the underlying one that enables determination of the coefficients $c_{\ell}$ given the electrical length $k L$ and the angle $\theta_{0}$ of the biconical antenna. To speed up computation of the quantities $g_{m l}$ in the series (32), it is possible to extract out the asymptotic tail of the series and rewrite it is

$$
\begin{aligned}
g_{m \ell}= & \sum_{n=1}^{\infty}\left\{\frac{\left(2 \nu_{n}+1\right)}{\nu_{n}\left(\nu_{n}+1\right)} \frac{\widehat{J}_{\nu_{n}}^{\prime}(k L)}{\widehat{J}_{\nu_{n}}(k L)} \frac{d \nu_{n}}{d \theta_{0}}\right. \\
& \frac{1}{\left[\nu_{n}\left(\nu_{n}+1\right)-\alpha_{0}^{2} \beta_{m}^{2}\right]\left[\nu_{n}\left(\nu_{n}+1\right)-\alpha_{0}^{2} \beta_{\ell}^{2}\right]} \\
& -\frac{1}{\pi \alpha_{0}^{2}\left(\beta_{m}+\beta_{\ell}\right) k L}\left[\frac{1}{\left(n-\beta_{m}\right)\left(n-\beta_{\ell}\right)}-\right. \\
& \left.\left.\frac{1}{\left(n+\beta_{m}\right)\left(n+\beta_{\ell}\right)}\right]\right\} \\
& +\frac{1}{\pi \alpha_{0}^{2}\left(\beta_{m}+\beta_{\ell}\right) k L}\left[\frac{\pi \sin \pi\left(\beta_{m}-\beta_{\ell}\right)}{\left(\beta_{m}-\beta_{\ell}\right) \sin \pi \beta_{m} \sin \pi \beta_{\ell}}+\right. \\
& \left.\frac{1}{\beta_{m} \beta_{\ell}}-2 \frac{\psi\left(\beta_{m}\right)-\psi\left(\beta_{\ell}\right)}{\beta_{m}-\beta_{\ell}}\right],
\end{aligned}
$$

where $\beta_{m}=\sqrt{m(m+1)} / \alpha_{0}$ and $\psi(x)=d \ln \Gamma(x) / d x$ is the Euler psi-function (a.k.a. digamma function) [19, 8.3601]. Note that 33 is still an exact expression but the series in (33) converges much faster than that in (32). As an example, for $\theta_{0}=\pi / 4, k L=\pi$, the terms within the series in (33) behave as $O\left(n^{-3.8}\right), n \gg 1$ for $m=5, \ell=9$.

The infinite linear system of equations contained in (31) can be cast in a matrix form as

$$
\mathcal{G} \mathbf{c}=\mathbf{p},
$$

where the vector $\mathbf{c}=\left[c_{1}, c_{3}, c_{5}, \ldots\right]^{T}$, the vector

$$
\mathbf{p}=j\left[P_{1}\left(\cos \theta_{0}\right), P_{3}\left(\cos \theta_{0}\right), P_{5}\left(\cos \theta_{0}\right), \ldots\right]^{T}
$$

and the symmetric matrix $\mathcal{G}=\left\{\mathcal{G}_{m \ell}\right\}$ has entries

$$
\begin{aligned}
\mathcal{G}_{m \ell}= & m(m+1) \ell(\ell+1) \sin \theta_{0} P_{m}\left(\cos \theta_{0}\right) P_{\ell}\left(\cos \theta_{0}\right) g_{m \ell} \\
& -\frac{m(m+1)}{2 m+1} \frac{\widehat{H}_{m}^{(2)^{\prime}}(k L)}{\widehat{H}_{m}^{(2)}(k L)} \delta_{m}^{\ell} .
\end{aligned}
$$

For a given angle $\theta_{0}$ and electrical length $k L$, the coefficients $c_{\ell}$ can be determined by solving the linear system (34). The coefficients $a_{\nu_{n}}$ are determined from these and (30). All components of fields are then completely known in regions I and II, but for the unimportant constant $I_{0}$.

Fig. 3 shows the normalized values of mode coefficients calculated from truncating the linear system $(34)$ to the first 20 odd modes (that is, $m \in(1,39)$ ) for $k L=\pi$ and $\theta_{0}=\pi / 4$. Clearly the coefficient strengths beyond $m=11$ diminish below $1 \%$ of the dominant mode. The real and imaginary parts of the actual mode coefficients are shown in Table II In general highly accurate results were found by considering $M_{i} \sim 10\lceil k L\rceil$ exterior modes so that the size of the truncated matrix $\mathcal{G}$ is of the order of $M_{i} \times M_{i}$. The series in (33) can also be truncated to $N \sim 10\lceil k L\rceil$.

The real and imaginary parts of the normalized input admittance of the antenna $K Y_{\text {in }}$ as calculated from (16), (17) and 290 are plotted in Fig. 4 as a function of $k L$ for $\theta_{0}=\pi / 4$. For this cone angle, the input admittance is seen to vary only slowly beyond $k L=1.25$, thus exhibiting wide-band behavior of the antenna for sufficiently long lengths. It is also seen that the frequency derivative of the susceptance, 


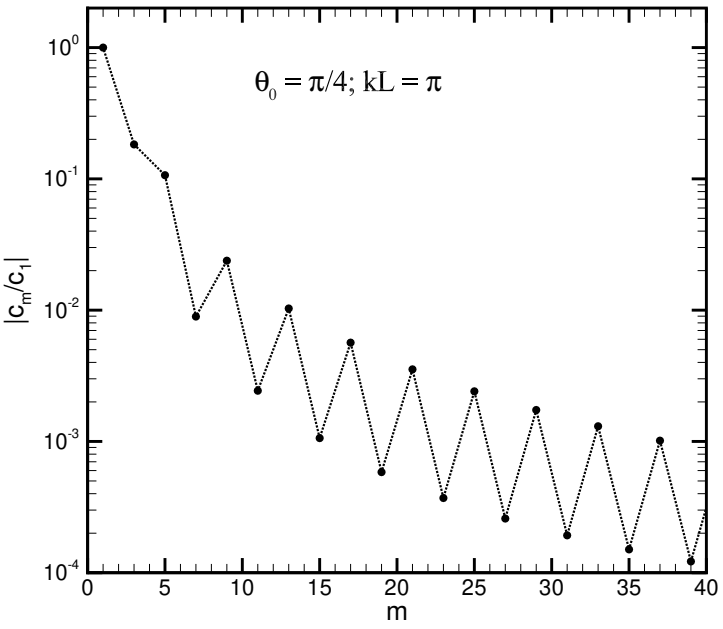

Fig. 3. The mode coefficients $\left|c_{m} / c_{1}\right|, m=1,3,5, \ldots$

TABLE II

Mode coefficients $c_{m}$ for $\theta_{0}=\frac{\pi}{4}, k L=\pi$.

\begin{tabular}{ccc}
\hline \hline Coefficient Index $m$ & Real $\left(c_{m}\right)$ & Imag $\left(c_{m}\right)$ \\
\hline 1 & $1.1490407 \mathrm{e}+00$ & $8.0338944 \mathrm{e}-02$ \\
3 & $-3.5425972 \mathrm{e}-02$ & $-2.0771627 \mathrm{e}-01$ \\
5 & $-4.0580443 \mathrm{e}-02$ & $-1.1596856 \mathrm{e}-01$ \\
7 & $-9.1395858 \mathrm{e}-03$ & $4.7392218 \mathrm{e}-03$ \\
9 & $9.0982654 \mathrm{e}-03$ & $2.5869270 \mathrm{e}-02$ \\
11 & $2.6832496 \mathrm{e}-03$ & $-8.1219646 \mathrm{e}-04$ \\
13 & $-4.1895639 \mathrm{e}-03$ & $-1.1097297 \mathrm{e}-02$ \\
15 & $-1.2124708 \mathrm{e}-03$ & $1.4597221 \mathrm{e}-04$ \\
17 & $2.3939406 \mathrm{e}-03$ & $6.0599842 \mathrm{e}-03$ \\
19 & $6.7224523 \mathrm{e}-04$ & $2.1939023 \mathrm{e}-05$ \\
21 & $-1.5394069 \mathrm{e}-03$ & $-3.7766908 \mathrm{e}-03$ \\
23 & $-4.2137034 \mathrm{e}-04$ & $-6.9357711 \mathrm{e}-05$ \\
25 & $1.0678991 \mathrm{e}-03$ & $2.5602905 \mathrm{e}-03$ \\
27 & $2.8676707 \mathrm{e}-04$ & $8.0535111 \mathrm{e}-05$ \\
29 & $-7.8122851 \mathrm{e}-04$ & $-1.8401158 \mathrm{e}-03$ \\
31 & $-2.0705664 \mathrm{e}-04$ & $-7.9689775 \mathrm{e}-05$ \\
33 & $5.9446707 \mathrm{e}-04$ & $1.3806263 \mathrm{e}-03$ \\
35 & $1.5633318 \mathrm{e}-04$ & $7.4903593 \mathrm{e}-05$ \\
37 & $-4.6631115 \mathrm{e}-04$ & $-1.0706105 \mathrm{e}-03$ \\
39 & $-1.2223804 \mathrm{e}-04$ & $-6.9109479 \mathrm{e}-05$ \\
\hline
\end{tabular}

$d\left[\Im\left(Y_{\text {in }}\right)\right] / d k$, is not a monotonous function of frequency. Fig. 4 shows that the frequency derivative is positive for $0.1<k L<0.7$ and $0.9<k L<2.5$. However, it turns negative for $0.7 \leq k L \leq 0.9$. These results confirm the conjecture [20] that Foster's reactance theorem [16, Ch. 8], which was developed for lossless passive networks does not hold even for perfectly conducting antennas. Such an invalidity of the Foster's reactance theorem to antennas was established analytically in [16, Sec. 8.1.3] for the case of an equatorial slot cut on the surface of a perfectly conducting sphere.

The E-plane variation of far-zone electric field $E_{\theta}$ and nearzone magnetic field $H_{\phi}(r=L, \theta)$ of the antenna with $k L=$ $2 \pi, \theta_{0}=\pi / 4$ are shown in Fig. 5. Both fields are normalized with respect to their maximum fields. The far-zone pattern for an electrically small biconical antenna resembles that of short dipole. However, for the pattern shown in Fig. 5 for a radius of $L / \lambda=1$, a single lobe broadside beam is observed for the biconical antenna in contrast to that of a two wavelength long cylindrical dipole, which will have split beams [14].

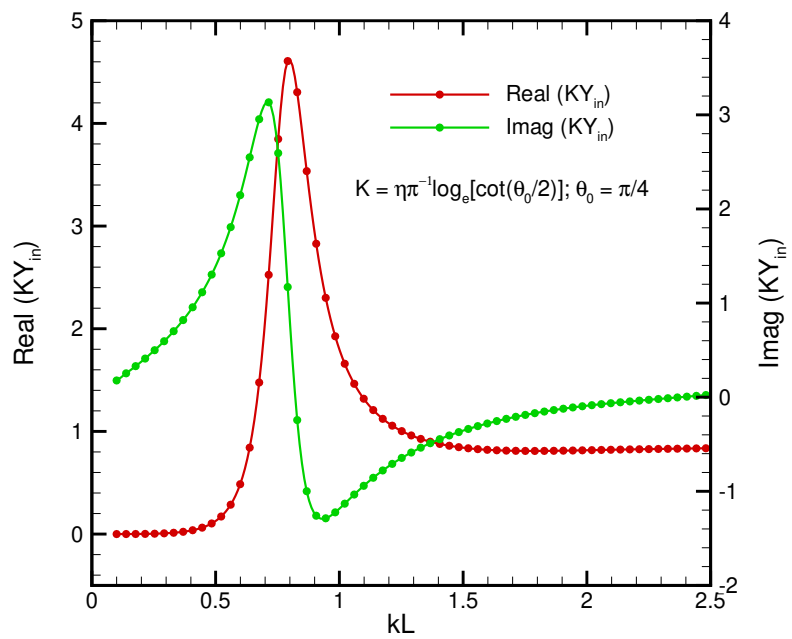

Fig. 4. Input admittance $K Y_{\text {in }}$ of the biconical antenna for $\theta_{0}=\pi / 4$.

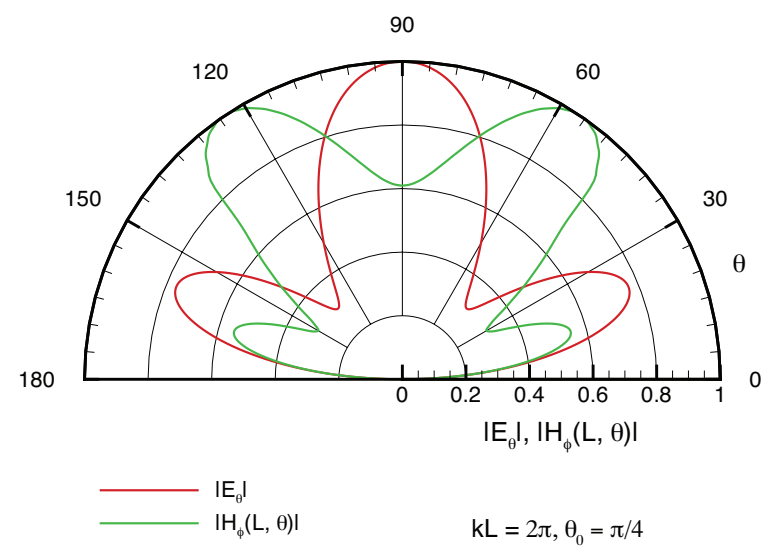

Fig. 5. Far-zone $\left|E_{\theta}(\theta)\right|$ and near-zone $\left|H_{\phi}(r=L, \theta)\right|$ of the biconical antenna for $\theta_{0}=\pi / 4, k L=2 \pi$.

\section{B. Quality Factor and Directivity}

Quality factor is an important metric in the understanding of the finite bandwidth behavior of an antenna. The quality factor, $Q$, of a lossless antenna is defined as $2 \pi$ times the ratio of effective energy, $W_{\text {sto }}$, stored in the fields to the energy radiated per cycle, $P_{\mathrm{rad}} \times T[21]$ and is

$$
Q=\frac{\omega W_{\text {sto }}}{P_{\text {rad }}},
$$


where $T=2 \pi / \omega$ is the RF time period. The radiated power $P_{\text {rad }}$ is

$$
P_{\mathrm{rad}}=\frac{1}{2} \iint_{\Omega}\left(r \mathbf{E} \times r \mathbf{H}^{*}\right) \cdot \hat{\mathbf{r}} d \Omega,
$$

where $\Omega$ denotes the unit sphere, and the effective stored energy is [22]

$W_{\text {sto }}=\frac{\epsilon_{0}}{4} \int_{r=0}^{\infty} d r \iint_{\Omega}\left(r^{2}\left[\mathbf{E} \cdot \mathbf{E}^{*}+\eta^{2} \mathbf{H} \cdot \mathbf{H}^{*}\right]-4 \eta P_{\text {rad }}\right) d \Omega$.

The radial integral in (39) can be split into two parts: $r \in$ $(0, L)$ plus $r \in(L, \infty)$, to correspond to the energy stored $W_{\text {sto }}(\mathrm{I})$ and $W_{\text {sto }}(\mathrm{II})$ in regions I and II, respectively.

Using the field expressions (3), (4) in (38) and utilizing (7), (9), and 27) gives

$$
\frac{2 \pi P_{\mathrm{rad}}}{\eta\left|I_{0}\right|^{2}}=: P_{o}=\frac{K^{2} \pi}{\eta} \Re\left(Y_{t}\right)=\sum_{\ell=1,3}^{\infty} \Re\left(c_{\ell}\right) P_{\ell}\left(\cos \theta_{0}\right) .
$$

Evidently, this is independent of the radius $r$. Fig. 6 shows the variation of the normalized radiated power $P_{o}$ with the electric length for a cone half-angle $\theta_{0}=\pi / 4$. The radiated power is very low for small electrical lengths and varies by four orders of magnitude as the length is varied over $k L \in(0.1,1.25)$.

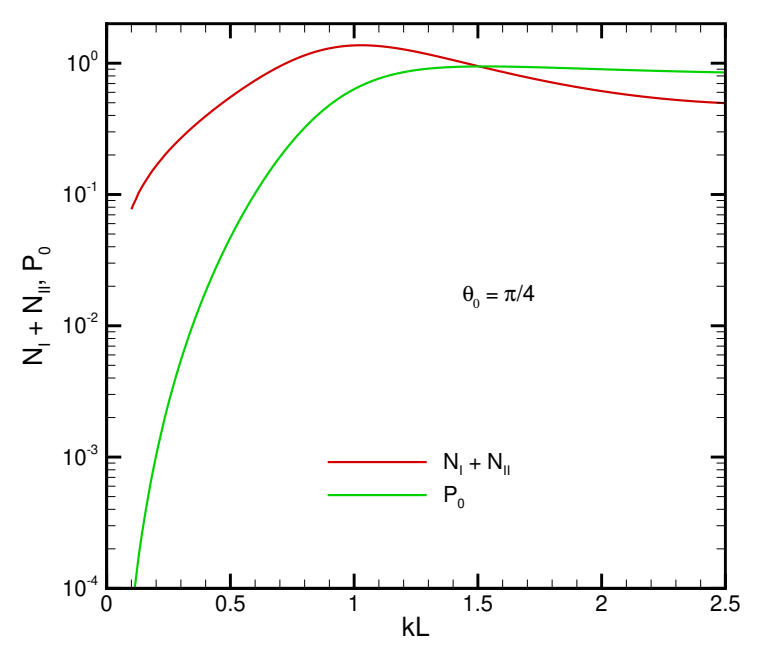

Fig. 6. Normalized effective power stored and power radiated by the biconical antenna for $\theta_{0}=\pi / 4$.

The radiated power could also be evaluated using the fields in region-II. Using equations (19), (20) in (38) and utilizing $\sqrt{25}$ and the Wronskian $W_{H}=\widehat{H}_{\ell}^{(2)}(z) \widehat{H}_{\ell}^{(1)}(z)-$ $\widehat{H}_{\ell}^{(1) \prime}(z) \widehat{H}_{\ell}^{(2)}(z)=-2 j$ gives

$$
P_{o}=\frac{2 \pi P_{\mathrm{rad}}}{\eta\left|I_{0}\right|^{2}}=\sum_{\ell=1,3}^{\infty} \frac{\ell(\ell+1)}{(2 \ell+1)} \frac{\left|c_{\ell}\right|^{2}}{\left|\widehat{H}_{\ell}^{(2)}(k L)\right|^{2}},
$$

which is once again independent of the radius $r$. It is easy to verify that the quantities on the RHSs of (40) and (41) are identical to each other by (i) starting from equation (31), multiplying both sides of it by $-j c_{m}$ and summing over all $m=1,3, \ldots \infty$,

(ii) taking the real part of the resulting summation,

(iii) and utilizing the symmetry relation $g_{m \ell}=g_{\ell m}$ and the Wronskian $W_{H}$.

Central to the evaluation of radiated power of a biconical antenna using either the interior fields or the exterior fields is the determination of the mode coefficients $c_{\ell}$ in the exterior region.

It is straightforward to evaluate the directivity, $D$, of the biconical antenna from expressions of the far-zone field given in 21 and the radiated power given in (41). The result is

$$
D=\frac{\max _{0<\theta<\pi}\left|\sum_{\ell=1,3}^{\infty} \frac{c_{\ell} j^{\ell}}{\widehat{H}_{\ell}^{(2)}(k L)} \frac{d P_{\ell}(\cos \theta)}{d \theta}\right|^{2}}{\sum_{\ell=1,3}^{\infty} \frac{\ell(\ell+1)}{(2 \ell+1)} \frac{\left|c_{\ell}\right|^{2}}{\left|\widehat{H}_{\ell}^{(2)}(k L)\right|^{2}}} .
$$

The directivity can be completely determined once the mode coefficients $c_{\ell}$ in the exterior field have been found out. Fig. 7 shows a plot of the directivity of the biconical antenna as a function of the electrical length for a fixed half-angle of $\theta_{0}=\pi / 4$. Also shown for comparison is the directivity of a filamentary cylindrical dipole having the same overall length. Both antennas have the same directivity of $D=1.5$ for very small electrical lengths. However, the beam begins to split at broadside in the region $1 / 4 \lesssim L / \lambda \lesssim 1 / 2$, in the case of a biconical antenna, thus resulting in reduced directivity compared to a cylindrical dipole. Nonetheless, the biconical antenna is seen to have much larger directivity bandwidth than a filamentary dipole for radii up to $L / \lambda \approx 1 / 4$.

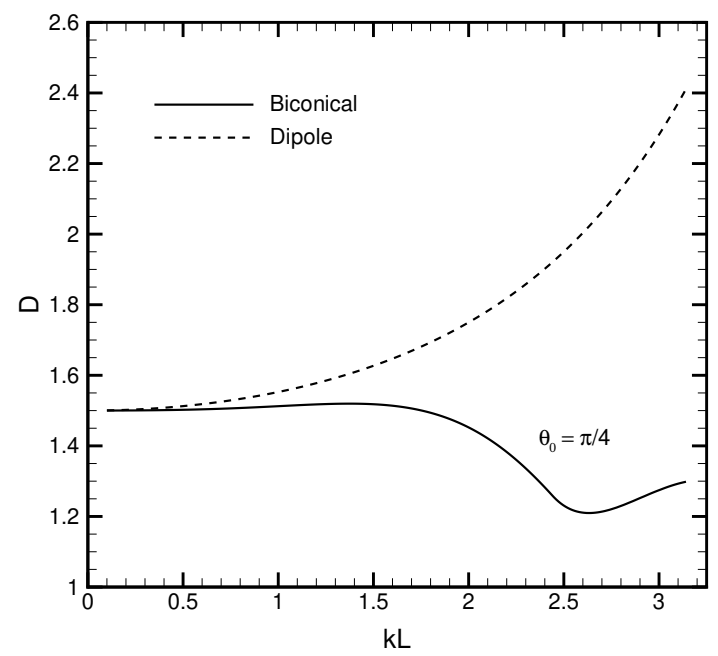

Fig. 7. Directivity of a biconical antenna for $\theta_{0}=\pi / 4$.

The effective energy stored by the antenna will be calculated separately in region-I $(0 \leq r<L)$ and region-II $(L \leq r<\infty)$ in accordance with the integral (39) and then added to yield the total energy stored in all space. It is convenient to use 
the expression 40 for $P_{\text {rad }}$ in the determination of $W_{\text {sto }}(\mathrm{I})$ and expression 41 in the determination of $W_{\text {sto }}$ (II). Using (2)-(4) and the results (28) and (27) it is easy to see that

$$
\begin{aligned}
& \iint_{\Omega} r^{2}\left|E_{r}\right|^{2} d \Omega=\frac{\left|I_{0}\right|^{2} \eta^{2} \sin \theta_{0}}{\pi(k r)^{2}} \sum_{n=1}^{\infty} \frac{\left|a_{\nu_{n}}\right|^{2}}{2 \nu_{n}+1} \\
& {\left[\frac{\widehat{J}_{\nu_{n}}(k r)}{\widehat{J}_{\nu_{n}}(k L)}\right]^{2} \frac{\left(\frac{\partial M_{\nu_{n}}}{\partial \theta_{0}}\right)^{2}}{\frac{d \nu_{n}}{d \theta_{0}}}} \\
& \iint_{\Omega} r^{2}\left|E_{\theta}\right|^{2} d \Omega=\frac{\left|I_{0}\right|^{2} \eta^{2} \sin \theta_{0}}{\pi} \sum_{n=1}^{\infty} \frac{\left|a_{\nu_{n}}\right|^{2}}{\nu_{n}\left(\nu_{n}+1\right)\left(2 \nu_{n}+1\right)} \\
& {\left[\frac{\widehat{J}_{\nu_{n}}^{\prime}(k r)}{\widehat{J}_{\nu_{n}}(k L)}\right]^{2} \frac{\left(\frac{\partial M_{\nu_{n}}}{\partial \theta_{0}}\right)^{2}}{\frac{d \nu_{n}}{d \theta_{0}}}+\frac{\left|I_{0}\right|^{2} \eta K}{2}[1+} \\
& K^{2}\left|Y_{t}\right|^{2}+\left(1-K^{2}\left|Y_{t}\right|^{2}\right) \cos 2 k(r-L) \\
& \left.+2 K \Im\left(Y_{t}\right) \sin 2 k(r-L)\right] \\
& \iint_{\Omega} r^{2}\left|\eta H_{\theta}\right|^{2} d \Omega=\frac{\left|I_{0}\right|^{2} \eta^{2} \sin \theta_{0}}{\pi} \sum_{n=1}^{\infty} \frac{\left|a_{\nu_{n}}\right|^{2}}{\nu_{n}\left(\nu_{n}+1\right)\left(2 \nu_{n}+1\right)} \\
& {\left[\frac{\widehat{J}_{\nu_{n}}(k r)}{\widehat{J}_{\nu_{n}}(k L)}\right]^{2} \frac{\left(\frac{\partial M_{\nu_{n}}}{\partial \theta_{0}}\right)^{2}}{\frac{d \nu_{n}}{d \theta_{0}}}+\frac{\left|I_{0}\right|^{2} \eta K}{2}[1+} \\
& K^{2}\left|Y_{t}\right|^{2}-\left(1-K^{2}\left|Y_{t}\right|^{2}\right) \cos 2 k(r-L) \\
& \left.-2 K \Im\left(Y_{t}\right) \sin 2 k(r-L)\right] \text {. }
\end{aligned}
$$

Adding these three quantities and extracting out the radiated power per (39), one gets after some simplifications the following integral for region-I

$$
\begin{gathered}
\int_{\zeta=0}^{k L} d \zeta \iint_{\Omega}\left(r^{2}\left[\mathbf{E} \cdot \mathbf{E}^{*}+\eta^{2} \mathbf{H} \cdot \mathbf{H}^{*}\right]-4 \eta P_{\mathrm{rad}}\right) d \Omega= \\
\frac{\left|I_{0}\right|^{2} \eta^{2} \sin \theta_{0}}{\pi} \sum_{n=1}^{\infty} \frac{\left|a_{\nu_{n}}\right|^{2}}{\left(2 \nu_{n}+1\right)} \frac{\left(\frac{\partial M_{\nu_{n}}}{\partial \theta_{0}}\right)^{2}}{\frac{d \nu_{n}}{d \theta_{0}}} \frac{1}{\widehat{J}_{\nu_{n}}^{2}(k L)} \times \\
\int_{0}^{k L}\left[\frac{\widehat{J}_{\nu_{n}}^{2}(\zeta)}{\zeta^{2}}+\frac{\widehat{J}_{\nu_{n}}^{2}(\zeta)}{\nu_{n}\left(\nu_{n}+1\right)}+\frac{\left[\widehat{J}_{\nu_{n}}^{\prime}(\zeta)\right]^{2}}{\nu_{n}\left(\nu_{n}+1\right)}\right] d \zeta \\
>0, \quad\left|I_{0}\right|^{2} \eta K\left|1-K Y_{t}\right|^{2} k L
\end{gathered}
$$

where the substitution $\zeta=k r$ was used. The integral involving Bessel functions can be evaluated in a closed form using integration by parts, followed by the identity [16, C.139] to finally result in the following expression for the normalized effective energy stored in region-I:

$$
\begin{aligned}
\frac{2 \pi \omega W_{\text {sto }}(\mathrm{I})}{\eta\left|I_{0}\right|^{2}=: N_{\mathrm{I}}=} & \frac{\sin \theta_{0}}{2} \sum_{n=1}^{\infty} \frac{\left|a_{\nu_{n}}\right|^{2}}{\left(2 \nu_{n}+1\right)} \frac{\left(\frac{\partial M_{\nu_{n}}}{\partial \theta_{0}}\right)^{2}}{\frac{d \nu_{n}}{d \theta_{0}}} \\
& {\left[\frac{k L}{\nu_{n}\left(\nu_{n}+1\right)}\left(1+\frac{\widehat{J}_{\nu_{n}}^{\prime 2}(k L)}{\widehat{J}_{\nu_{n}}^{2}(k L)}\right)-\frac{1}{k L}\right] } \\
& +\frac{\pi K}{2 \eta} k L\left|1-K Y_{t}\right|^{2} .
\end{aligned}
$$

For $k L \rightarrow 0$, asymptotic forms of Bessel functions can be used to result in the asymptotic expression

$$
N_{\mathrm{I}} \sim \frac{\sin \theta_{0}}{2 k L} \sum_{n=1}^{\infty} \frac{\left|a_{\nu_{n}}\right|^{2}}{\nu_{n}\left(2 \nu_{n}+1\right)} \frac{\left(\frac{\partial M_{\nu_{n}}}{\partial \theta_{0}}\right)^{2}}{\frac{d \nu_{n}}{d \theta_{0}}}, k L \rightarrow 0 .
$$

Note that the mode coefficients $a_{\nu_{n}}$ are themselves dependent on $k L$ through the coefficients $c_{\ell}$ as is evident from (30) and 31. Note also that one can avoid the explicit calculation of the quantity $\partial M_{\nu_{n}} / \partial \theta_{0}$ that is needed in (44). It is indeed clear from 30, and (28) that $\sin \theta_{0}\left|a_{\nu_{n}}\right|^{2}\left(\partial M_{\nu_{n}} / \partial \theta_{0}\right)^{2} /\left[d \nu_{n} / d \theta_{0}\right]=$ $\nu_{n}\left(\nu_{n}+1\right)\left(2 \nu_{n}+1\right) \sum_{\ell} \sum_{m} c_{\ell} c_{m}^{*} m(m+1) \ell(\ell+$ 1) $T_{\ell, \nu_{n}}\left(\theta_{0}\right) T_{m, \nu_{n}}\left(\theta_{0}\right) / I_{\nu_{n}, \nu_{n}}\left(\theta_{0}\right)$ and the ratio $T_{\ell, \nu_{n}}\left(\theta_{0}\right) T_{m, \nu_{n}}\left(\theta_{0}\right) / I_{\nu_{n}, \nu_{n}}\left(\theta_{0}\right)$ is independent of $\partial M_{\nu_{n}} / \partial \theta_{0}$. The stored power inside region-I, a positive definite quantity, is completely determined once the mode coefficients $c_{\ell}$ in the exterior region have been found.

In a similar fashion using the expressions (18)-(20) for the fields in region-II and the identities (24) and (25) it can be shown that with $\zeta=k r$

$$
\begin{array}{r}
r^{2} \iint_{\Omega}\left[\mathbf{E} \cdot \mathbf{E}^{*}+\eta^{2} \mathbf{H} \cdot \mathbf{H}^{*}\right] d \Omega=\frac{\left|I_{0}\right|^{2} \eta^{2}}{\pi} \\
\sum_{\ell=1,3}^{\infty} \frac{\ell(\ell+1)\left|c_{\ell}\right|^{2}}{(2 \ell+1)\left|\widehat{H}_{\ell}^{(2)}(k L)\right|^{2}} \times \\
{\left[\ell(\ell+1) \frac{\left|\widehat{H}_{\ell}^{(2)}(\zeta)\right|^{2}}{\zeta^{2}}+\left|\widehat{H}_{\ell}^{(2) \prime}(\zeta)\right|^{2}+\left|\widehat{H}_{\ell}^{(2)}(\zeta)\right|^{2}\right] .}
\end{array}
$$

From this and the identities [16, C.139, C.154], the expression for the normalized effective stored energy in region-II is obtained as

$$
\begin{aligned}
\frac{2 \pi \omega W_{\text {sto }}(\mathrm{II})}{\eta\left|I_{0}\right|^{2}}=: N_{\mathrm{II}}= & \frac{k L}{2} \sum_{\ell=1,3}^{\infty} \frac{\ell(\ell+1)\left|c_{\ell}\right|^{2}}{(2 \ell+1)\left|\widehat{H}_{\ell}^{(2)}(k L)\right|^{2}} \times \\
& {\left[\left(\frac{\ell(\ell+1)}{(k L)^{2}}-1\right)\left|\widehat{H}_{\ell}^{(2)}(k L)\right|^{2}+\right.} \\
& 2-\mid \widehat{H}_{\ell}^{\left.\left.(2){ }^{\prime}(k L)\right|^{2}\right] .}
\end{aligned}
$$

Note that $N_{\text {II }}>0$ for any $k L$ in view of the inequality [16. C.148]. The sum of $N_{\mathrm{I}}$ and $N_{\text {II }}$ is thereby a positive definite quantity for any $k L$ and $\theta_{0}$. 
In terms of the normalized stored energies and the radiated power, the quality-factor of the antenna is

$$
Q=\frac{\left(N_{\mathrm{I}}+N_{\mathrm{II}}\right)}{P_{o}} \text {. }
$$

The sum of two stored energy quantities $N_{\mathrm{I}}$ and $N_{\mathrm{II}}$ for $\theta_{0}=$ $\pi / 4$ is also plotted in Fig. 6. It is seen that the dynamic range in the variation of the sum is far less than that of the radiated power. Fig. 8 shows the $Q$-factor and the inverse $Q$-factor for the biconical antenna with $\theta_{0}=\pi / 4$. Comparison is shown with the Chu's limit of $Q_{\text {chu }}=(k L)^{-1}\left[1+(k L)^{-2}\right]$ [22]. It is interesting to see that the $Q$-factor of a biconical antenna stays very close to the lower limit imposed by Chu. A high $Q$ and the high slope at smaller antenna lengths is a consequence of the relatively low radiated power as evident from Figure 6

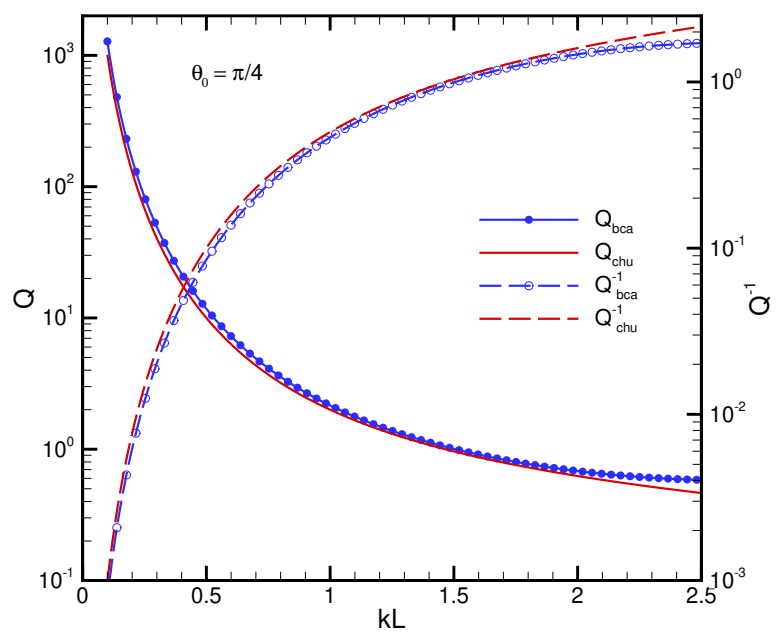

Fig. 8. Quality factor of a biconical antenna for $\theta_{0}=\pi / 4$. Comparison is shown with Chu's limit $Q_{\mathrm{chu}}=(k L)^{-1}\left[1+(k L)^{-2}\right]$.

\section{CONCLUSION}

Complete expressions have been provided for the analysis of a biconical antenna of arbitrary cone angle and length. Numerical data have been generated for various quantities for a cone angle of $\theta_{0}=\pi / 4$. A wide-angle biconical antenna has many desirable features and the following steps summarize the key findings of the study:

(i) Equations (11) and (12) provide highly accurate analytical expressions for the roots $\nu_{n}\left(\theta_{0}\right)$ and the derivative $d \nu_{n} / d \theta_{0}$.

(ii) The mode coefficients $c_{\ell}$ are determined from the matrix equation (34) and (35) with the matrix entries of $\mathcal{G}$ filled from 36 and 33 .

(iii) The input admittance $Y_{\text {in }}$ of the antenna is determined from (16), (17) and 29). Numerical results for the admittance variation for $\theta_{0}=\pi / 4$ indicate that the antenna is broadband for radii $L / \lambda \gtrsim 1 / 4$. Furthermore, susceptance data in Fig. 4 clearly demonstrate that Foster's reactance theorem remains invalid even for perfectly conducting antennas. (iv) The directivity, $D$, of the antenna is determined from 42). Results for $\theta_{0}=\pi / 4$ indicate that the directivity of the antenna is a slowly varying function of its electrical length and departs significantly from that of a filamentary cylindrical dipole having the same electrical length.

(v) The quality factor $Q=\left(N_{\mathrm{I}}+N_{\mathrm{II}}\right) / P_{o}$ of the antenna is determined from (41), (44), and (46). Expression for $Q$ includes energy stored in the fields both in the interior and exterior of the circumscribing sphere. Results for $\theta_{0}=\pi / 4$ demonstrate that the quality factor of the biconical antenna is very close to the lower limit established by Chu.

\section{REFERENCES}

[1] S. A. Schelkunoff, "Principal and complementary waves in antennas," Proc. IRE, vol. 34, pp. 23-32, 1946.

[2] C. T. Tai, "On the theory of biconical antennas," J. Applied Physics, vol. 19, pp. 1155-1159, December 1948.

[3] S. A. Schelkunoff, Advanced Antenna Theory. New York, NY: John Wiley \& Sons, Inc., 1952.

[4] R. E. Collin and F. J. Zucker, Antenna Theory, Pt. 1, ser. Inter-University Electronics Series. New York, NY: Mc-Graw-Hill Book Co., 1969, vol. 7.

[5] C. T. Tai, Antenna Engineering Handbook. New York, NY: Mc-GrawHill Book Co., 1984, ch. Ch. 4: Dipoles and Monopoles.

[6] C. H. Papas and R. W. P. King, "Input impedance of wide-angle conical antennas fed by a coaxial line," Proc. IRE, vol. 37, pp. 1269-1271, 1949.

[7] —_, "Radiation from wide-angle conical antennas fed by a coaxial line," Proc. IRE, vol. 39, pp. 49-51, 1951.

[8] R. M. Bevensee, Handbook of Conical Antennas and Scatterers. New York: Gordon and Breach, 1973.

[9] P. E. Mayes and W. Gee, "Tunable, wide-angle conical monopole with selectable bandwidth," in Proceedings of the 1995 Antenna Applications Symposium. Hanscom AFB, MA: Rome Laboratory, 1995.

[10] S. N. Samaddar and E. L. Mokole, "Biconical antennas with unequal cone angles," IEEE Trans. Antennas Propagat., vol. 46, no. 2, pp. 181192, November 1998.

[11] S. Z. Sapuan, A. Kazemipour, and M. Jenu, "Direct feed biconical anenna as a reference antenna." Seremban, Malaysia: 2011 IEEE International RF and Micorwave Conference (RFM 2011), 12-14 December 2011.

[12] R. Janaswamy, "Biconical antenna: A wideband benchmark antenna for IEEE P2816." Marina Bay Sands, Singapore: 2021 IEEE International Symposium on Antennas and Propagation and USNC-URSI Radio Science Meeting, 4-10 December 2021.

[13] W. L. Stutzman and G. A. Thiele, Antenna Theory and Design, 3rd ed. Hoboken, NJ: John Wiley \& Sons, Inc., 2013.

[14] C. A. Balanis, Advanced Engineering Electromagnetics, 2nd ed. New York: John Wiley \& Sons, Inc., 2012.

[15] J. M. Jin, Theory and Computation of Electromagnetic Fields, 2nd ed. New York, NY: John Wiley \& Sons, Inc., 2015.

[16] R. Janaswamy, Engineering Electrodynamics: A collection of theorems, principles and field representations. Bristol, U.K.: Institute of Physics, December 2020.

[17] S. A. Schelkunoff, Applied Mathematics for Engineers and Scientists. Princeton, NJ: Van Nostrand, 1965.

[18] N. N. Lebedev, Special Functions and Their Applications. New York, NY: Dover Publications, 1972.

[19] I. S. Gradshteyn and I. M. Ryzhik, Tables of Integrals, Series and Products, 7th ed. Academic Press, 2007.

[20] M. Capek and L. Jelinek, "Comments on 'stored energy and radiation Q', IEEE Trans. Antennas Propagat., vol. 64, pp. 4575-4576, 2016.

[21] IEEE Antennas \& Propagation Standards Committee, IEEE P145: Standard for Definition of Terms for Antennas, Institute of Electrical and Electronics Engineers., Inc., 3 Park Avenue, New York, New York 10016-5997, 2020.

[22] R. E. Collin and S. Rothschild, "Evaluation of antenna Q," IEEE Trans. Antennas Propagat., vol. 12, pp. 23-27, January 1964. 